Short Communication

\title{
A novel c.1037C > G (p.Ala346Gly) mutation in TP63 as cause of the ectrodactyly-ectodermal dysplasia and cleft lip/palate (EEC) syndrome
}

\author{
Leandro Ucela Alves ${ }^{1}$, Eliete Pardono ${ }^{2}$, Paulo A. Otto ${ }^{1}$ and Regina Célia Mingroni Netto ${ }^{1}$ \\ ${ }^{1}$ Departamento de Genética e Biologia Evolutiva, Instituto de Biociências, \\ Universidade de São Paulo, São Paulo, SP, Brazil. \\ ${ }^{2}$ Faculdade de São Paulo, Grupo Educacional UNIESP, São Paulo, SP, Brazil.
}

\begin{abstract}
Ectrodactyly - ectodermal dysplasia and cleft lip/palate (EEC) syndrome (OMIM 604292) is a rare disorder determined by mutations in the TP63 gene. Most cases of EEC syndrome are associated to mutations in the DNA binding domain (DBD) region of the $\mathrm{p} 63$ protein. Here we report on a three-generation Brazilian family with three individuals (mother, son and grandfather) affected by EEC syndrome, determined by a novel mutation c.1037C > G (p.Ala346Gly). The disorder in this family exhibits a broad spectrum of phenotypes: two individuals were personally examined, one presenting the complete constellation of EEC syndrome manifestations and the other presenting an intermediate phenotype; the third affected, a deceased individual not examined personally and referred to by his daughter, exhibited only the split-hand/foot malformation (SHFM). Our findings contribute to elucidate the complex phenotype-genotype correlations in EEC syndrome and other related TP63-mutation syndromes. The possibility of the mutation c.1037C > G being related both to acro-dermato-ungual-lacrimal-tooth (ADULT) syndrome and SHFM is also raised by the findings here reported.
\end{abstract}

Keywords: EEC syndrome, TP63-mutations, p63-associated disorders, SHFM.

Received: May 5, 2014; Accepted: September 10, 2014.

Ectrodactyly-ectodermal dysplasia and cleft lip/palate (EEC) syndrome (OMIM 604292) is a rare autosomaldominant disorder characterized by dry or eczematous skin, sparse hair on the scalp, eyebrows and eyelashes, nail dystrophy and hypodontia, in addition to ectrodactyly and orofacial cleft. Other clinical manifestations include syndactyly, absence or reduced number of sweat, sebaceous, and salivary glands, mammary gland/nipple hypoplasia, nasolacrimal duct abnormalities, conductive or sensorineural hearing loss, facial dysmorphisms, chronic infections of the respiratory tract, genitourinary anomalies, and development delay (Clements et al., 2009; Celli et al., 1999; Shivaprakash et al., 2012). EEC is a complex pleiotropic condition of multiple congenital dysplasia and anomalies. The clinical diagnosis of the syndrome is hampered by its extremely variable expression (Rodini and RichieriCosta, 1990; Celli et al., 1999; Rinne et al., 2006). The penetrance is practically complete and just a few cases of arguable lack of penetrance have been reported (Roelfsema and Cobben, 1996).

Send correspondence to Regina Célia Mingroni-Netto. Departamento de Genética e Biologia Evolutiva, Instituto de Biociências, Universidade de São Paulo, Rua do Matão 277, 05508090 São Paulo, SP, Brazil. E-mail: renetto@ib.usp.br.
Most EEC cases, if not all, result from mutations in the TP63 gene, and more than 200 cases have already been reported in the literature (Clements et al., 2009). EEC is one out of five distinct ectodermal dysplasia (ED) syndromes caused by mutations in this gene, which include, besides EEC, acro-dermato-ungual-lacrimal-tooth syndrome (ADULT, OMIM 103285), ankyloblepharon-ectodermal defects-cleft lip/palate syndrome (AEC, OMIM 106260), limb-mammary syndrome (LMS, OMIM 603543), and Rapp-Hodgkin syndrome (RHS, OMIM 129400). Nonsyndromic split hand/foot malformation (SHFM4, OMIM 605289) and, very rarely, nonsyndromic cleft lip may be caused by mutations in this same TP63 gene (Leoyklang et al., 2006; Rinne et al., 2006). All conditions related to TP63 mutations exhibit clinical features that largely overlap with EEC syndrome, complicating the differential diagnosis among them (Clements et al., 2009; Celik et al., 2011).

The protein $\mathrm{p} 63$, encoded by TP63 gene on $3 \mathrm{q} 27$, is a member of p53 family. Like the TP53 and TP73 genes, TP63 encodes a sequence-specific transcription factor that activates target genes involved in different cellular pathways. However, unlike the $\mathrm{p} 53$ protein, $\mathrm{p} 63$ protein is not involved in tumor suppression, but plays an important role as a transcription factor involved in limb, epithelial, and 
craniofacial development (Rinne et al., 2007; van Bokhoven et al., 2011). p63 is expressed as at least six different isoforms, three of which include a transactivation domain (TAp63) and the other three, none $(\Delta \mathrm{Np} 63)$. Alternative splicing produces additional three $\mathrm{C}$-terminus regions, $\alpha, \beta$ and $\gamma$ isoforms. $\Delta \mathrm{Np} 63 \alpha$ is the major isoform expressed in the epidermis (Browne et al., 2011). The EEC syndrome is mainly caused by missense mutations in the DNA binding domain (DBD), a region with transactivation activity present in all isoforms (Celli et al., 1999; Rinne et al., 2007).

Here we report a novel heterozygous missense mutation in TP63 (c.1037C > G) that results in p.Ala346Gly, causing EEC in a Brazilian family (Figure 1).

The proband, a woman born in the Northern region of Brazil, presents the complete manifestation of the EEC syndrome (Figure 2), bilateral split hand and split foot malformation (SHFM), cleft lip and palate, dracryocystitis (secondary to obstruction of the lacrimal duct) with corneal ulceration, blepharitis, blepharophimosis, bilateral sensorineural hearing loss, hypodontia (Figure 2A, C and E). In addition, she had hypothyroidism.

Her affected son also had bilateral SHFM. In the lower limbs, the splitting was accompanied by malformation of the leg bones, probably tibial and/or fibular hemimelia (lower limb extremities and distal region of the leg bones were amputated and no X-rays were available). He also presented dracryocystitis, hypodontia, thin and hypopigmented hair, blepharitis, and blepharophimosis, but no labiopalatine cleft (Figure 2B and D).

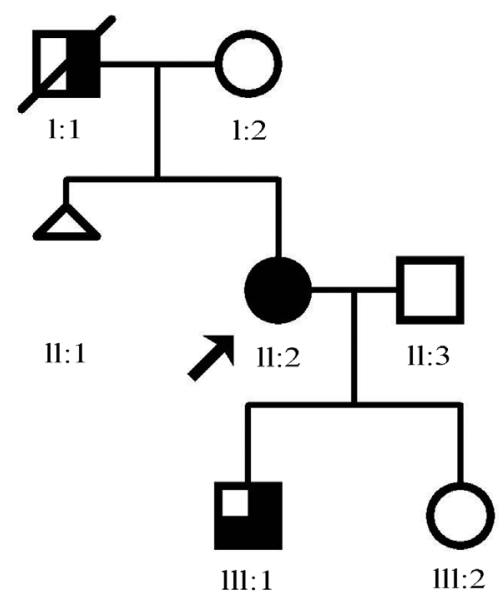

Ectrodactyly

Ectrodactyly, ectodermal dysplasia and cleft lip/palate

Ectrodactyly and ectodermal dysplasia

Figure 1 - Pedigree of family with ectrodactyly-ectodermal dysplasia and cleft lip/palate (EEC) syndrome.
The deceased father of the proband was reported to have only SHFM, without cleft lip/palate or other features associated with the syndrome.

Blood samples were obtained with informed consent from the proband and her children. Genomic DNA was extracted from peripheral blood leukocytes using standard techniques. Initially, the DNA was amplified by PCR using primers that flank the entire coding sequence of the TP63 gene. The amplified product was sequenced bi-directionally using the BigDye Terminator v3.1 Cycle Sequencing Kit method in an ABI 3730 DNA Analyzer equipment (Applied Biosystems, Foster City, CA, USA). Sequencing of both strands was performed at least twice for each sample.

The results were analyzed by the Bioedit program (Ibis Biosciences, Carlsbad, CA, USA). Prediction of the effect of the mutation was performed with the Polyphen-2, SIFT and MutationTaster2 online programs. The protein alignment analysis was done using the Universal Protein Resource (Uniprot) catalog available online. The mutation was also searched in the databases of the 1000 Genomes and the NHLBI Exome Sequencing Project. The numbering of nucleotides and amino acids used in this report is in accordance with systematic nomenclature approved by the Human Genome Variation Society (HGVS).

Molecular analysis of the TP63 gene identified a novel heterozygous missense change c.1037C $>\mathrm{G}$ in exon 8 , present in the proband and in her affected son. It was absent in her unaffected daughter. This mutation has never been described before and is not present in the 1000 Genomes and NHLBI exome sequencing project databases. The mutation c. $1037 \mathrm{C}>\mathrm{G}$ in TP63 that results in a p.Ala346Gly amino acid substitution, was predicted to be probably damaging by PolyPhen-2 (score of 0,997 ) and SIFT (score of 0), and to be disease causing by MutationTaster2. The protein sequence alignment showed that alanine at position 346 lies in the DBD region and is highly conserved across different species (Uniprot). The TP63 mutation here described has been submitted to the ClinVar Database (SCV000189629). More than 30 different mutations have been described in the literature, and only two of them are located outside the DBD region (Celli et al., 1999; Rinne et al., 2006; Clements et al., 2009).

Mutations in the DBD region were reported to disrupt the DNA-binding ability of p63, which results in reduced transactivation activity (Celli et al., 1999). p63 protein plays an important role in the development, proliferation, and maintenance of stratified epithelia, such as the epidermis (Koster et al., 2004). Mice lacking Tp63 display defects in epithelial lineage development and fail to develop stratified epithelia and epithelial appendages (Mills et al., 1999; Yang et al., 1999), which explains the skin, glands, teeth and hair defects in the EEC syndrome.

Irf6, one of p63 target genes, is needed for skin differentiation and palate development, including palate closure (Moretti et al., 2010). Mutations in Irf6 were associated 


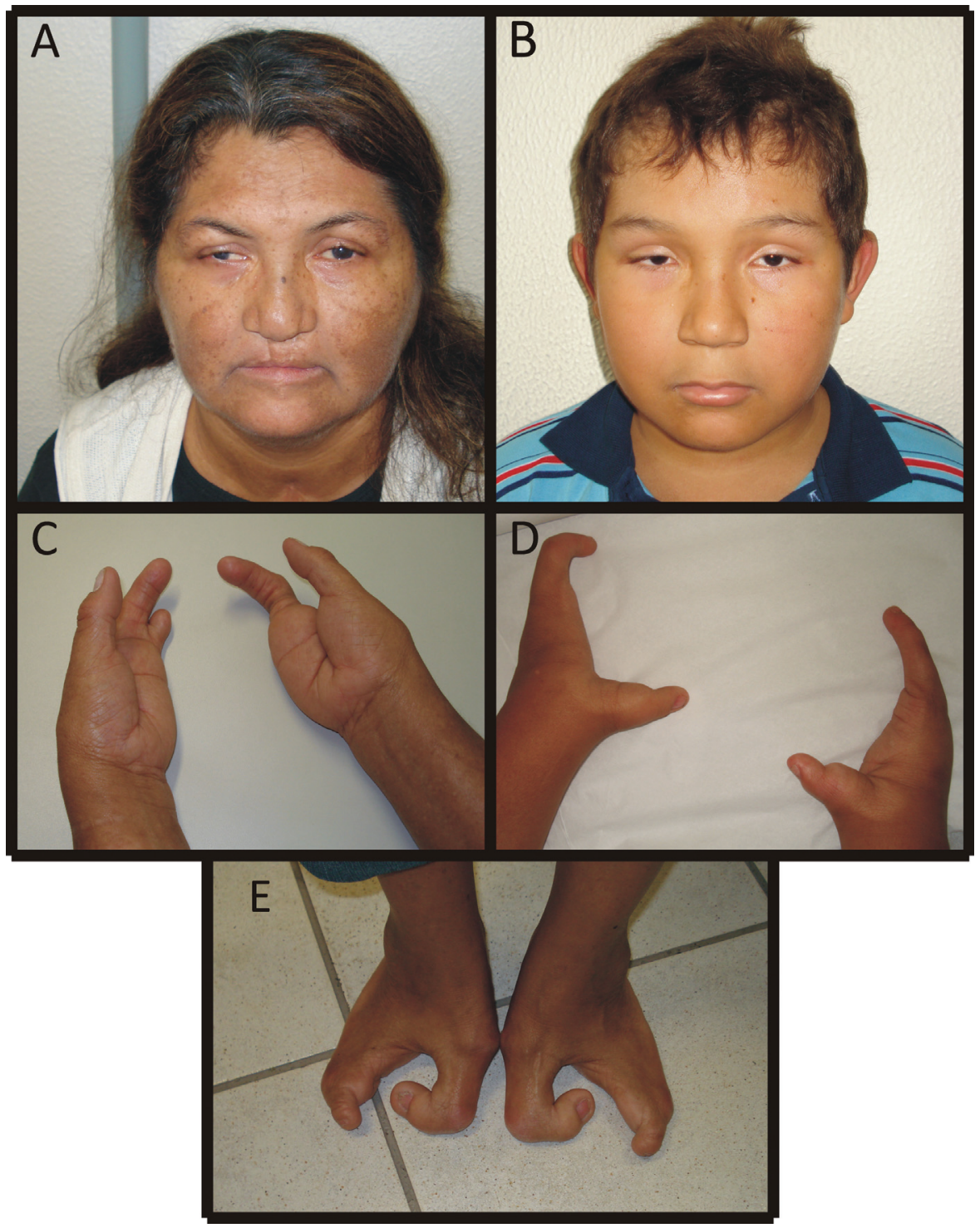

Figure 2 - Images showing split-hand/foot malformation (SHFM) in patients investigated. (A) proband; (B) proband's son; (C) hands of proband; (D) hands of proband's son; (E) feet of proband.

with cleft lip/palate and other developmental disorders in mice (Richardson et al., 2006). A feedback regulatory loop between Irf6 and p63 was suggested (Guerrini et al., 2011), and, therefore, mutations in TP63 could explain orofacial cleft.

The apical ectodermal ridge (AER) on limb-bud, which is responsible for limb development, is maintained by self-renewing stem cells. It was speculated that $\mathrm{p} 63$ has an essential role in preserving self-renewal capacity of progenitor epithelial cells (Yang et al., 1999). Moreover, there is evidence that p63 is able to induce the transcription of Dlx 5 and Dlx6 promoters in the AER (Lo Iacono et al., 2008), and the DLX5 gene was already associated with SHFM (type I) in humans (Shamseldin et al., 2012). Double knockout mice for Dlx5 and Dlx6 present SHFM (Merlo et al., 2002). Restelli et al. (2014) proposed a model for a regulatory loop during limb development. According to their model: p63 activates Dlx5/Dlx6 transcription; Dlx5/Dlx6 positively controls Fgf8; p63 also activates Fgf8 transcription; Pin1 (peptidil-prolyl cis/trans isomerase NIMA-interacting-1) induces $\mathrm{p} 63$ destabilization mediated by proteasome degradation; on the other hand, Fgf8 acts on the AER cells and promotes p63 stability, apparently because it protects p63 from Pin-1 interaction. The activation of this loop and maintenance of $\mathrm{p} 63$ levels allows stratification and specialization of ectoderm cells in the AER. These findings, taken together, can explain the clinical features of isolated forms of SHFM and SHFM in EEC syndrome.

In defects associated to TP63 mutations, genotypephenotype-correlations are hampered by extremely variable expressivity both among and within families (Rinne $e t$ al., 2007; Di Lorio et al., 2012). The situation is further 
complicated by the fact that affected individuals by (apparently) different diseases associated with the TP63 gene may be carriers of identical mutations: such is the case of the mutation p.R243W associated with EEC (Celli et al., 1999) and ADULT (Avitan-Hersh et al., 2010) syndromes, and the case of the mutation p.R319H, that can cause the EEC syndrome (van Bokhoven et al., 2001) as well as nonsyndromic SHFM (Ianakiev et al., 2000).

This variable expressivity of EEC is evident in the present study: besides the proband's son not having cleft lip/palate, features present in his mother, his leg malformation is much more severe when compared to the one presented by his mother. Since the proband's son does not have cleft lip/palate, his phenotype is similar to that of ADULT syndrome, which may not include clefting, breast hypoplasia or freckling (Berk et al., 2012). The significant clinical overlap between the two phenotypes demonstrates that the distinctions between EEC and ADULT syndromes are somewhat artificial, as already pointed out by Reisler et al. (2006). The father of the proband allegedly presented only SHFM malformation affecting his four limbs, without other manifestations of EEC. This could indicate that the mutation c. $1037 \mathrm{C}>\mathrm{G}$ might also be associated with nonsyndromic SHFM, in spite of a certain degree of uncertainty due to the fact that this individual is already deceased and could not be personally examined by one of us.

As already stressed, this is the first report of the mutation c.1037C > G (p.Ala346Gly). Clements et al. (2009) found a mutation at the same amino acid position, a change from alanine to asparagine (p. Ala346Asp) (in their report the mutation was formerly described as p.Ala307Asp), which caused the EEC syndrome in two patients of the same family, mother and son. They both displayed hypodontia and bilateral SHFM. The ectodermal anomalies were similar in mother and son. Some differences between the patients from his and our report were observed in the clefting pattern, since it was bilateral in the case of p.Ala346Asp mutation and unilateral in ours. Another important difference between these two reports concerns the large spectrum of clinical variability observed within families: while the patients studied by Clements et al. (2009) differed only in relation to the presence of lacrimal ducts dysplasia, our patients differed in relation to the presence of cleft lip/palate and to the variability spectrum of limb malformation.

The present findings contribute to elucidate the complex phenotype-genotype correlations in EEC syndrome and other related TP63-mutation syndromes. The possibility of the mutation c. $1037 \mathrm{C}>\mathrm{G}$ being related to both ADULT syndrome and SHFM is also raised by the findings here reported.

\section{Acknowledgments}

We thank Mrs. Maria Teresa Balester de Mello Auricchio for technical assistance. We also thank CEPID-
FAPESP and CAPES for the financial support and the patients for their cooperation.

\section{References}

Avitan-Hersh E, Indelman M, Bergman R and Sprecher E (2010) ADULT syndrome caused by a mutation previously associated with EEC syndrome. Pediatr Dermatol 27:643-645.

Berk DR, Armstrong NL, Shinawi M and Whelan AJ (2012) ADULT syndrome due to an R243W mutation in TP63. Int J Dermatol 51:693-696.

Browne G, Cipollone R, Lena AM, Serra V, Zhou H, van Bokhoven H, Dötsch V, Merico D, Mantovani R, Terrinoni A, et al. (2011) Differential altered stability and transcriptional activity of $\Delta$ Np63 mutants in distinct ectodermal dysplasias. J Cell Sci 124(Pt 13):2200-2207.

Celik TH, Buyukcam A, Simsek-Kiper PO, Utine GE, ErsoyEvans S, Korkmaz A, Yntema HG, Bodugroglu K and Yurdakok M (2011) A newborn with overlapping features of AEC and EEC syndromes. Am J Med Genet A 155A:31003103.

Celli J, Duijf P, Hamel BC, Bamshad M, Kramer B, Smits AP, Newbury-Ecob R, Hennekam RC, Van Buggenhout G, van Haeringen A, et al. (1999) Heterozygous germline mutations in the p53 homolog p63 are the cause of EEC syndrome. Cell 99:143-153.

Clements SE, Techanukul T, Coman D, Mellerio JE and McGrath JA (2009) Molecular basis of EEC (ectrodactyly, ectodermal dysplasia, clefting) syndrome: five new mutations in the DNA-binding domain of the TP63 gene and genotypephenotype correlation. Br J Dermatol 162:201-207.

Di Lorio E, Kaye SB, Ponzin D, Barbaro V, Ferrari S, Böhm E, Nardiello P, Castaldo G, McGrath JA and Willoughby CE (2012) Limbal stem cell deficiency and ocular phenotype in ectrodactyly-ectodermal dysplasia-clefting syndrome caused by p63 mutations. Ophthalmology 119:74-83.

Guerrini L, Costanzo A and Merlo GR (2011) A symphony of regulations centered on p63 to control development of ectoderm-derived structures. J Biomed Biotechnol 2011:864904

Ianakiev P, Kilpatrick MW, Toudjarska I, Basel D, Beighton P and Tsipouras P (2000) Split-hand/split-foot malformation is caused by mutations in the p63 gene on $3 q 27$. Am J Hum Genet 67:59-66.

Koster MI, Kim S, Mills AA, DeMayo FJ and Roop DR (2004) p63 is the molecular switch for initiation of an epithelial stratification program. Genes Dev 18:126-131.

Leoyklang P, Siriwan P and Shotelersuk V (2006) A mutation of the p63 gene in non-syndromic cleft lip. J Med Genet 43:e28.

Lo Iacono N, Mantero S, Chiarelli A, Garcia E, Mills AA, Morasso MI, Costanzo A, Levi G, Guerrini L and Merlo GR (2008) Regulation of Dlx 5 and Dlx6 gene expression by p63 is involved in EEC and SHFM congenital limb defects. Development 135:1377-1388.

Merlo GR, Paleari L, Mantero S, Genova F, Beverdam A, Palmisano GL, Barbieri O and Levi G (2002) Mouse model of split hand/foot malformation type I. Genesis 33:97-101.

Mills AA, Zheng B, Wang XJ, Vogel H, Roop DR and Bradley A (1999) p63 is a p53 homologue required for limb and epidermal morphogenesis. Nature 398:708-713. 
Moretti F, Marinari B, Lo Iacono N, Botti E, Giunta A, Spallone G, Garaffo G, Vernersson-Lindahl E, Merlo G, Mills AA, et al. (2010) A regulatory feedback loop involving p63 and IRF6 links the pathogenesis of 2 genetically different human ectodermal dysplasias. J Clin Invest 120:1570-1577.

Reisler TT, Patton MA and Meagher PP (2006) Further phenotypic and genetic variation in ADULT syndrome. Am J Med Genet A 140:2495-2500.

Restelli M, Lopardo T, Lo Iacono N, Garaffo G, Conte D, Rustighi A, Napoli M, Del Sal G, Perez-Morga D, Costanzo A, et al. (2014) DLX5, FGF8 and the Pin1 isomerase control $\triangle \mathrm{Np} 63 \alpha$ protein stability during limb development: a regulatory loop at the basis of the SHFM and EEC congenital malformations. Hum Mol Genet 23:3830-3842.

Richardson RJ, Dixon J, Malhotra S, Hardman MJ, Knowles L, Boot-Handford RP, Shore P, Whitmarsh A and Dixon MJ (2006) Irf6 is a key determinant of the keratinocyte proliferation-differentiation switch. Nat Genet 38:1329-1334.

Rinne T, Brunner HG and van Bokhoven H (2007) p63-associated disorders. Cell Cycle 6:262-268.

Rinne T, Hamel B, van Bokhoven H and Brunner HG (2006) Pattern of p63 mutations and their phenotypes - Update. Am J Med Genet A 140:1396-1406.

Rodini ES and Richieri-Costa A (1990) EEC syndrome: report on 20 new patients, clinical and genetic considerations. Am J Med Genet 37:42-53.

Roelfsema NM and Cobben JM (1996) The EEC syndrome: a literature study. Clin Dysmorphol 5:115-127.

Shamseldin HE, Faden MA, Alashram W and Alkuraya FS (2012) Identification of a novel $D L X 5$ mutation in a family with autosomal recessive split hand and foot malformation. $\mathrm{J}$ Med Genet 49:16-20.

Shivaprakash PK, Joshi HV, Noorani H and Reddy V (2012) Ectrodactyly, ectodermal dysplasia, and cleft lip/palate syndrome: A case report of "Incomplete syndrome". Contemp Clin Dent 3(Suppl 1):S115-S117.

van Bokhoven H, Hamel BC, Bamshad M, Sangiorgi E, Gurrieri F, Duijf PH, Vanmolkot KR, van Beusekom E, van Beersum SE, Celli J, et al. (2001) p63 gene mutations in eec syn- drome, limb-mammary syndrome, and isolated split handsplit foot malformation suggest a genotype-phenotype correlation. Am J Hum Genet 69:481-492.

van Bokhoven H, Melino G, Candi E and Declercq W (2011) p63, a story of mice and men. J Invest Dermatol 131:1196-1207.

Yang A, Schweitzer R, Sun D, Kaghad M, Walker N, Bronson RT, Tabin C, Sharpe A, Caput D, Crum C, et al. (1999) p63 is essential for regenerative proliferation in limb, craniofacial and epithelial development. Nature 398:714-718.

\section{Internet Resources}

1000 genomes, http://www.1000genomes.org/ (accessed January $15,2014)$

ClinVar, http://www.ncbi.nlm.nih.gov/clinvar/intro/ (accessed October 9, 2014).

Human Genome Variation Society (HGVS), http://www.hgvs.org/mutnomen (accessed October 15, 2013).

MutationTaster2, http://www.mutationtaster.org/ (accessed July 01, 2014).

NHLBI Exome Sequencing Project (ESP): Exome Variant Server, http://evs.gs.washington.edu/EVS/ (accessed July 01, 2014).

OMIM: online mendelian inheritance in man, http://www.omim.org/ (accessed January 15, 2014).

PolyPhen-2: prediction of functional effects of human nsSNPs, http://genetics.bwh.harvard.edu/pph2/ (accessed December 20, 2013).

SIFT: sorting Tolerant From Intolerant, http://sift.jcvi.org/ (accessed July 01, 2014).

Uniprot: universal protein resource, http://www.uniprot.org/ (accessed December 20, 2013).

Associate Editor: Patrícia Ashton-Prolla

License information: This is an open-access article distributed under the terms of the Creative Commons Attribution License, which permits unrestricted use, distribution, and reproduction in any medium, provided the original work is properly cited. 